\title{
Stage IIIC Ovarian Cancer AJCC v6 and v7
}

National Cancer Institute

\section{Source}

National Cancer Institute. Stage IIIC Ovarian Cancer A/CC v6 and v7. NCI Thesaurus.

Code C6260.

Stage IIIC includes: (T3c, N0, M0); (Any T, N1, M0). T3c: Peritoneal metastasis beyond pelvis more than $2 \mathrm{~cm}$ in greatest dimension and/or regional lymph node metastasis. NO:

No regional lymph node metastasis. N1: Regional lymph node metastasis. M0: No distant metastasis. (AJCC 6th and 7th eds.) 